\title{
Fine-tuning of the initial conditions for hybrid inflation
}

\author{
N. Tetradis \\ CERN, Theory Division, CH-1211, Geneva 23, Switzerland \\ (Received 28 July 1997; revised manuscript received 20 January 1998; published 7 April 1998)
}

\begin{abstract}
We study the evolution of regions of space with various initial field values for a simple theory that can support hybrid inflation. Only very narrow domains within the range of initial field values below the Planck scale lead to the onset of inflation. This implies a severe fine-tuning for the initial configuration that will produce inflation. [S0556-2821(98)02010-4]
\end{abstract}

PACS number(s): $98.80 . \mathrm{Cq}$

One of the most attractive features of hybrid inflation [1] is that it can take place for values of the inflation field below the Planck scale [2]. This permits the construction of realistic field theoretical models without the need of a description of quantum gravity. Several such models have been proposed within the context of supersymmetry [2-9]. Inflation requires the presence of an almost flat direction in field space with a nonzero vacuum energy. A very small slope along this direction results in the slow rolling of the inflation field $\phi$. For hybrid inflation, the slow rolling occurs along a valley of the effective potential. It ends when the valley turns into a ridge and the slow-roll regime terminates, owing to the growth of fluctuations of fields orthogonal to the inflation field. Typically this point of instability corresponds to a value $\phi_{\text {ins }}$ of the inflation below the Planck scale.

The question we would like to address is whether generic initial values of the fields below the Planck scale can lead to the onset of inflation. Clearly, there is an area where this is likely, the almost flat direction. Our aim is to explore the whole field space below the Planck scale and establish whether inflation is a frequent occurrence or a rare event. Similar investigations have already been performed in Refs. $[10,11]$. Our main observation is that the field range that can lead to inflation is much more constrained than what one would naively infer from Refs. [10, 11], unless a significant fine-tuning of the fields is imposed within the space regions that eventually inflate.

The onset of inflation requires a region of space, the size of a few Hubble lengths, where the fields take almost constant values, so that the gradient energy density is negligible compared to the potential energy density. The earliest time at which one could start talking about such regions of space is when the Planck era (during which quantum gravitational fluctuations dominate) ends and classical general relativity starts becoming applicable. The initial field values within each region are expected to be of order $M_{\mathrm{Pl}}$, or larger if the couplings of the theory are small, as in chaotic inflation [12]. In this work we concentrate on field values below $M_{\mathrm{Pl}}$ for two reasons: (a) Hybrid inflation does not require extremely small couplings. (b) In general, the field-theoretical models for which a potential can be reliably computed assume an expansion in powers of $\phi / M_{\mathrm{Pl}}$.

Inflation could start at the end of the Planck era if the fields take values in the parts of the potential that are able to support it. However, there is also the possibility that the fields will evolve, from some initial values that do not give inflation, to different values that do. We consider all possible field values, assuming an initial degree of homogeneity over a few Hubble lengths, and follow the evolution by integrating the classical equations of motion. In this way we determine the range of field values that eventually lead to inflation. We show that, for many realistic models, the initial fields must be extremely homogeneous if their initial values are below the Planck scale. For example, for the prototype model of hybrid inflation (see below), one of the fields must be homogeneous with at least $\sim 10^{-6}-10^{-5}$ accuracy. It is very difficult to calculate a probability distribution for the initial field configurations, and thus have a quantitative estimate of the probability of inflation to occur. This requires a reliable description of Planck scale dynamics. For this reason we do not address the question of whether such a homogeneous initial state is probable or not. Our results have a much looser interpretation, such as indicating how "natural" hybrid inflation is.

We consider the simplest potential that can support hybrid inflation [1]

$$
V(\phi, \sigma)=\frac{1}{4} \lambda\left(\sigma^{2}-M^{2}\right)^{2}+\frac{1}{2} m^{2} \phi^{2}+\frac{1}{2} g \phi^{2} \sigma^{2},
$$

with positive mass terms and couplings. It possesses the symmetries $\phi \rightarrow-\phi$ and $\sigma \rightarrow-\sigma$. A renormalizable term $\lambda^{\prime} \phi^{4}$ is permitted by these symmetries and should naturally appear. However, neglecting such a term can be justified if the hybrid inflationary scenario is embedded in the context of supersymmetry and an appropriate superpotential is chosen. The term $m^{2} \phi^{2} / 2$ is also absent in supersymmetric models, and the small slope along the inflationary trajectory is generated by nonrenormalizable (such as logarithmic) terms that are present when quantum corrections are taken into account $[3,5,9]$.

We use the simplest choice of Eq. (1) for our study. Our results do not depend on whether the slope along the inflationary trajectory is generated by the term $m^{2} \phi^{2} / 2$ or radiative corrections. In the concluding paragraphs we discuss a simple superpotential to which our results apply directly. We point out, however, that our analysis must be repeated for the determination of the appropriate initial conditions in supersymmetric inflationary models with more complicated superpotentials.

The potential of Eq. (1) has a valley of minima along the $\phi$ axis $(\sigma=0)$ for fixed $\phi^{2}>\phi_{\text {ins }}^{2}$, where 


$$
\phi_{\text {ins }}= \pm \sqrt{\frac{\lambda}{g}} M .
$$

There is a series of maxima along the $\phi$ axis $(\phi=0)$ for fixed $\phi^{2}<\phi_{\text {ins. }}^{2}$. The absolute minima of the potential are located at $(\phi=0, \sigma= \pm M)$. We assume that a RobertsonWalker metric is a good approximation for the regions of space with uniform fields that we are considering. The evolution of the fields is given by the standard equations

$$
\begin{gathered}
H^{2}=\left(\frac{\dot{R}}{R}\right)^{2}=\frac{8 \pi}{3 M_{\mathrm{Pl}}^{2}}\left[\frac{1}{2} \dot{\phi}^{2}+\frac{1}{2} \dot{\sigma}^{2}+V(\phi, \sigma)\right], \\
\ddot{\phi}+3 H \dot{\phi}=-\frac{\partial V(\phi, \sigma)}{\partial \phi}=-m^{2} \phi-g \sigma^{2} \phi, \\
\ddot{\sigma}+3 H \dot{\sigma}=-\frac{\partial V(\phi, \sigma)}{\partial \sigma}=\lambda M^{2} \sigma-\lambda \sigma^{3}-g \phi^{2} \sigma,
\end{gathered}
$$

with $M_{\mathrm{Pl}}=1.2 \times 10^{19} \mathrm{GeV}$.

Inflation occurs along the $\phi$ axis, where the potential is

$$
V(\phi)=\frac{1}{4} \lambda M^{4}+\frac{1}{2} m^{2} \phi^{2} .
$$

In the limit where the second term in the right-hand side (RHS) of the above equation dominates, we obtain the prototype model of chaotic inflation. This takes place for values of the field $\phi^{2} \gg M_{\mathrm{Pl}}^{2}$. The Cosmic Background Explorer (COBE) observation of the cosmic microwave background anisotropy leads to the constraint $m \simeq 10^{-6} M_{\mathrm{Pl}}$ [12]. As we have already explained, we are interested in values of the field $\phi^{2} \lesssim M_{\mathrm{Pl}}^{2}$, for which the first term in the RHS of Eq. (6) dominates during inflation. This occurs for

$$
2 m^{2} M_{\mathrm{Pl}}^{2} \ll \lambda M^{4} \text {. }
$$

The "slow-roll"' parameters [13] are given by [2]

$$
\begin{aligned}
\eta(\phi) & =\frac{M_{\mathrm{Pl}}^{2}}{8 \pi} \frac{d^{2} V(\phi) / d \phi^{2}}{V(\phi)}=\frac{m^{2} M_{\mathrm{Pl}}^{2}}{2 \pi\left(\lambda M^{4}+2 m^{2} \phi^{2}\right)} \\
& \simeq \frac{1}{2 \pi} \frac{m^{2} M_{\mathrm{Pl}}^{2}}{\lambda M^{4}}, \\
\epsilon(\phi) & =\frac{M_{\mathrm{Pl}}^{2}}{16 \pi}\left(\frac{d V(\phi) / d \phi}{V(\phi)}\right)^{2}=\frac{M_{\mathrm{Pl}}^{2} m^{4} \phi^{2}}{\pi\left(\lambda M^{4}+2 m^{2} \phi^{2}\right)^{2}} \\
& =4 \pi \eta^{2} \frac{\phi^{2}}{M_{\mathrm{Pl}}^{2}} .
\end{aligned}
$$

In the interval $\phi_{\text {ins }}^{2} \leqslant \phi^{2} \leqslant M_{\mathrm{Pl}}^{2}$ the conditions for inflation $\epsilon, \eta \ll 1$ are automatically satisfied for the parameter range of Eq. (7).

The number of $e$-foldings that occur between the moment that cosmologically interesting scales (of the order of the present Hubble distance) leave the horizon and the end of inflation is $N \simeq 60$. We denote by $\phi_{60}$ the value of the inflation field at horizon crossing for these scales. In our model, inflation terminates when $\phi$ rolls beyond the instability point $\phi_{\text {ins }}$. In the parameter range of Eq. (7), the relation between $\phi_{60}$ and $\phi_{\text {ins }}$ is [2]

$$
\phi_{60}^{2} \simeq \phi_{\mathrm{ins}}^{2} e^{120 \eta},
$$

with $\eta$ given by Eq. (8). The inflationary prediction for the spectrum of adiabatic density perturbations at cosmological scales $[14,13]$ is

$$
\delta_{H}^{2}=\frac{32}{75} \frac{V_{60}}{M_{\mathrm{Pl}}^{4}} \frac{1}{\epsilon_{60}} \simeq \frac{8 \pi}{75} \frac{\lambda^{3} M^{12}}{M_{\mathrm{Pl}}^{6} m^{4} \phi_{60}^{2}} .
$$

Comparison with the value $\delta_{H}=1.94 \times 10^{-5}$, deduced from the COBE observation of the cosmic microwave background anisotropy, leads to the constraint [2]

$$
\frac{M}{M_{\mathrm{Pl}}} \simeq 0.13 e^{12 \eta} g^{-1 / 10} \lambda^{-1 / 5}\left(\frac{m}{M_{\mathrm{Pl}}}\right)^{2 / 5} .
$$

For couplings that are not many orders of magnitude smaller than 1 and a sufficiently small "slow-roll" parameter $\eta$, we obtain a simple relation between the scales $M$ and $m$ :

$$
\frac{M}{M_{\mathrm{Pl}}} \simeq 0.13\left(\frac{m}{M_{\mathrm{Pl}}}\right)^{2 / 5} .
$$

The above relation holds as long as our assumption that the first term in the RHS of Eq. (6) dominates is valid. As we have already mentioned, when the second term dominates, this model reduces to the prototype model of chaotic inflation, for which the COBE observations imply $m$ $\simeq 10^{-6} M_{\mathrm{Pl}}$. We expect, therefore, that Eq. (13) holds as long as $m \lesssim 10^{-6} M_{\mathrm{Pl}}$. This leads to the constraint $M \lesssim 5$ $\times 10^{-4} M_{\mathrm{Pl}}$. A detailed analysis [2] verifies this conclusion and predicts a more accurate bound $M \leqq 2 \times 10^{-3} M_{\mathrm{Pl}}$. A similar bound applies to all models of hybrid inflation with couplings of order 1 . On general grounds one expects the inflationary energy scale $V^{1 / 4}$ to be at least two or three orders of magnitude smaller than the Planck scale [15]:

$$
V^{1 / 4} / \epsilon^{1 / 4} \simeq 7 \times 10^{16} \mathrm{GeV} .
$$

In order to determine the range of field values that lead to inflation, we start by considering a region of space, the size of a few Hubble lengths, in which the fields are homogeneous. We determine the evolution of this region by integrating Eqs. (3)-(5) numerically. We consider field values 0 $\leqslant \phi, \sigma \leqslant M_{\mathrm{Pl}}$, and couplings $\lambda=g=1$. The choice of couplings has a small effect on our conclusions, as long as they are not much smaller than 1 . Since we are interested in the necessary conditions for the onset of inflation, we neglect the term $-m^{2} \phi$ in Eq. (4) during the numerical integration. We assume that inflation occurs if the evolution stops on the $\phi$ axis beyond the instability point. If there is a significant slope along the $\phi$ axis, the range of field values that lead to inflation is smaller than our predictions.

In Fig. 1 we display an array of initial field values in Planck units for a theory with $M=10^{-1} M_{\mathrm{Pl}}$. The dark hexagons denote initial conditions that lead to inflation along the positive $\phi$ axis. The trajectories are short, with the $\phi$ field moving directly to its final value, while the $\sigma$ field performs damped oscillations around zero. The white hexagons denote noninflationary trajectories that end up at the minimum at $(\phi=0, \sigma=M)$. The white triangles denote trajectories that end up at the minimum at $(\phi=0, \sigma=-M)$. The white 


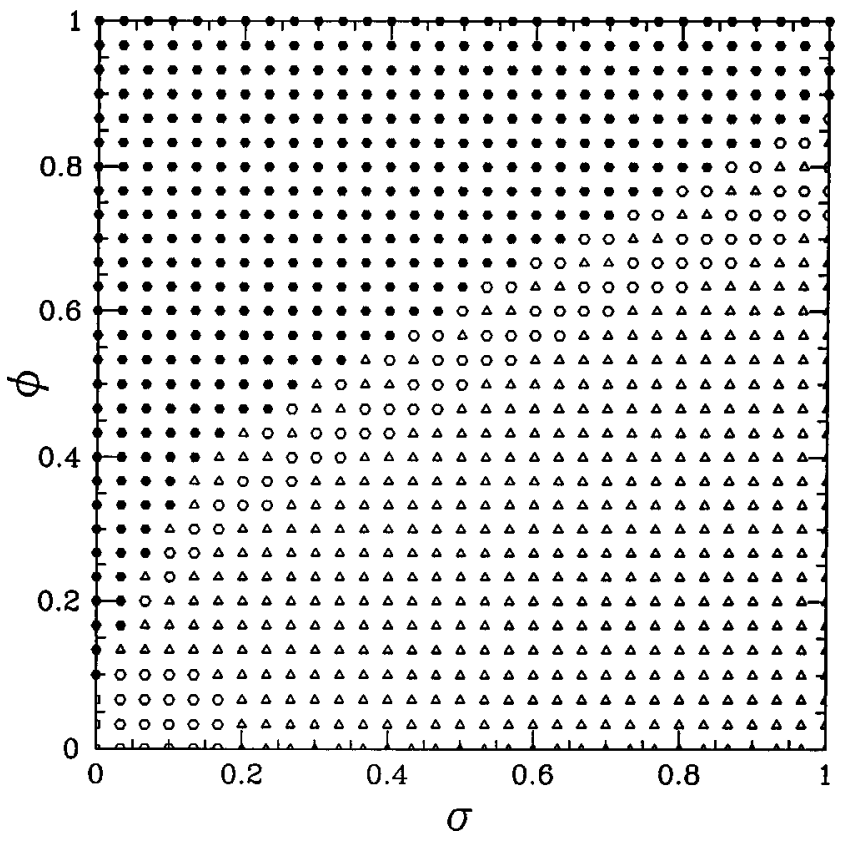

FIG. 1. $M=10^{-1} M_{\mathrm{Pl}}, \lambda=1, g=1$.

squares at the beginning of the $\phi$ axis denote noninflationary trajectories that start below the instability point $(\phi$ $=\sqrt{\lambda / g} M, \sigma=0)$ and end up at one of the two minima through the growth of fluctuations of the $\phi$ field. We observe a large continuous range of initial conditions that lead to the onset of inflation. This range starts at the instability point.

In Fig. 2 we display a similar array of initial field values for $M=3 \times 10^{-2} M_{\mathrm{Pl}}$. The black triangles indicate initial values that lead to inflation along the negative $\phi$ axis. We observe that the large continuous range of inflationary initial values has moved towards the Planck scale. A thin strip remains along the $\phi$ axis that ends at the instability point. Trajectories starting in the rest of field space rarely lead to the onset of inflation. Both fields oscillate around zero before

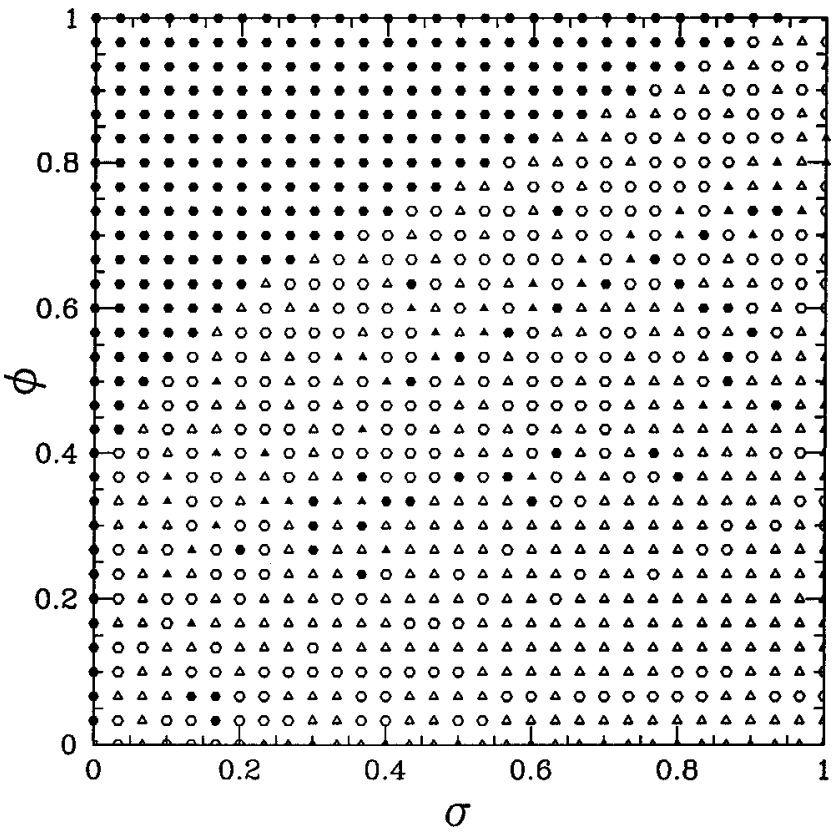

FIG. 2. $M=3 \times 10^{-2} M_{\mathrm{Pl}}, \lambda=1, g=1$.

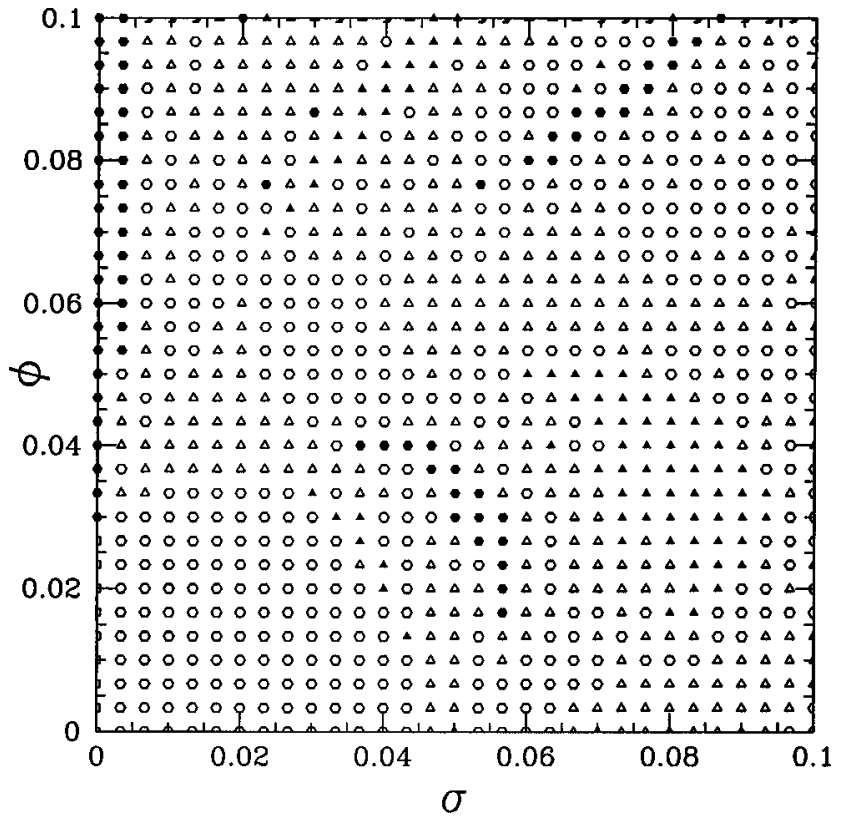

FIG. 3. Magnification of Fig. 2.

the system relaxes either at one of the minima or in the valleys along the $\phi$ axis. In Fig. 3 we present a magnification of Fig. 2 around the origin, which demonstrates the size of the various areas that lead to inflation. The largest of these has a size $\sim 2-3 \times 10^{-2} M_{\mathrm{Pl}}$, but the typical size is $\sim 10^{-2} M_{\mathrm{Pl}}$. The thin strip around the $\phi$ axis has a width $\sim 5 \times 10^{-3} M_{\mathrm{Pl}}$. Another magnification of Fig. 2, away from the origin, is presented in Fig. 4. Inflationary strips of width $\sim 10^{-2} M_{\mathrm{Pl}}$ are visible.

Another array of initial field values, for $M=10^{-2} M_{\mathrm{Pl}}$, is presented in Fig. 5. The situation is similar to that of Fig. 2, but the large inflationary range has moved further towards the Planck scale. Figures 6 and 7 are magnifications of Fig. 5 that demonstrate the size of the various areas. The inflationary strips of Fig. 6 have a width $\sim 1-2 \times 10^{-6} M_{\mathrm{Pl}}$. Both

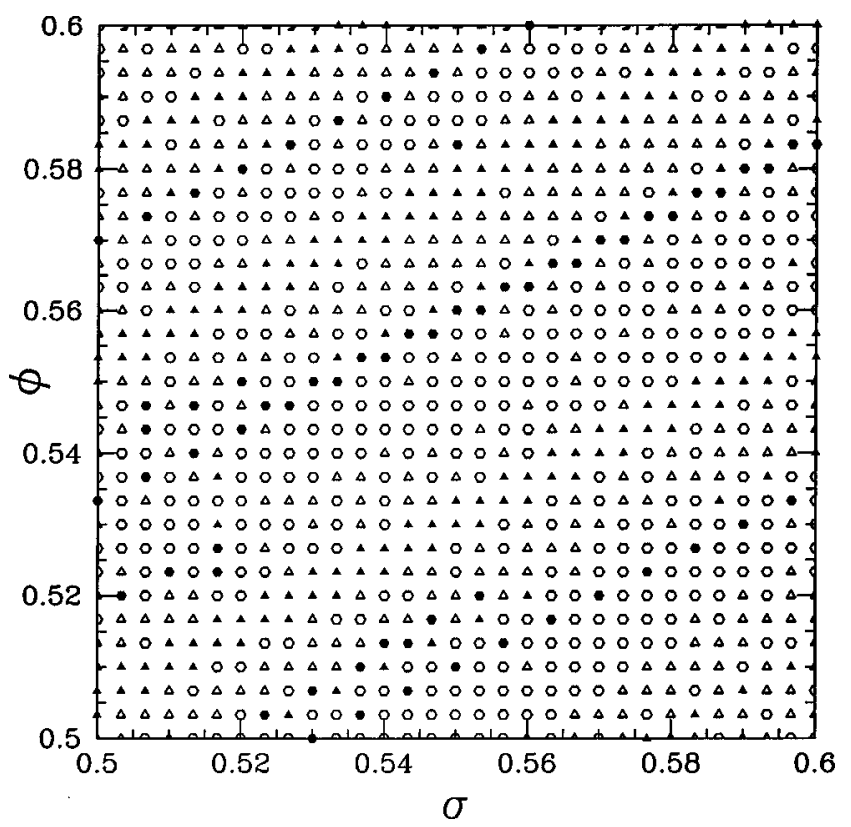

FIG. 4. Magnification of Fig. 2. 


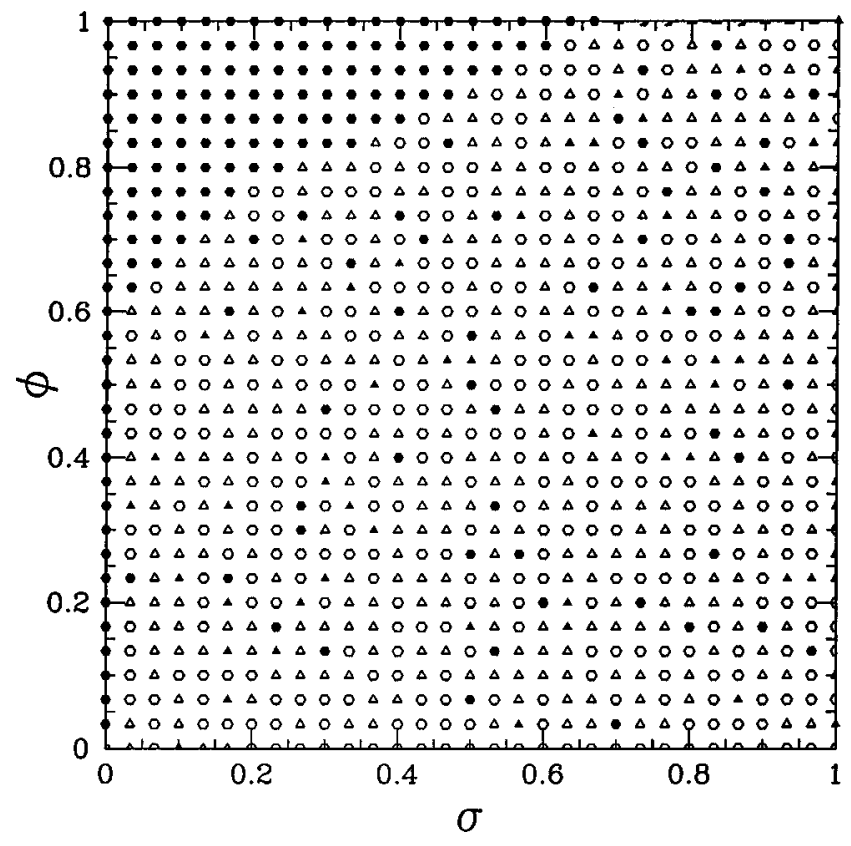

FIG. 5. $M=10^{-2} M_{\mathrm{Pl}}, \lambda=1, g=1$.

fields oscillate many times before the evolution stops. The strip along the $\phi$ axis has a width $\sim 4 \times 10^{-4} M_{\mathrm{Pl}}$.

The shrinking of the areas of field space that lead to inflation is due to the decrease of $H$ during the last stages of the evolution as $M$ is lowered. This reduces the magnitude of the friction term in Eqs. (4) and (5). Consequently, it takes longer for the system to settle down either at the minima or in the valleys along the $\phi$ axis. Many oscillations of the fields are required before most of the initial potential energy is dissipated through expansion. This results in increasing sensitivity to the initial conditions. A slight change of the initial field values causes the system to move off an inflationary trajectory and end up at one of the minima instead. For $M \leqslant 10^{-2} M_{\mathrm{Pl}}$ the largest area that leads to inflation, for

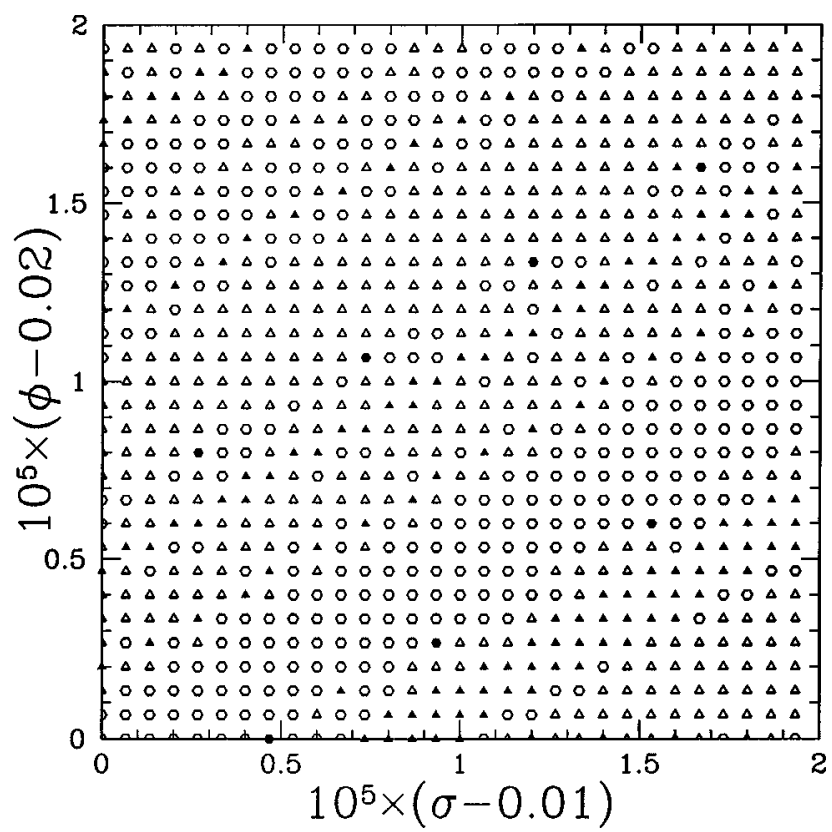

FIG. 6. Magnification of Fig. 5.

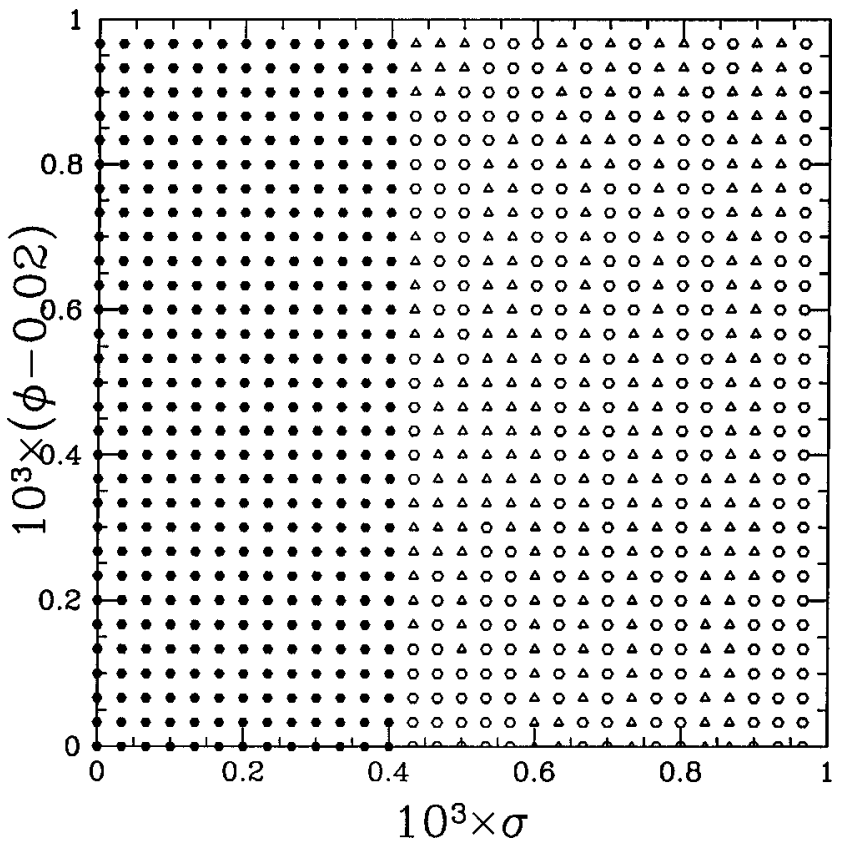

FIG. 7. Magnification of Fig. 5.

initial field values not very close to the Planck scale, is the strip along the $\phi$ axis. There, the evolution is rather simple, with only the $\sigma$ field oscillating and $\phi$ moving directly to its final value.

We can obtain an estimate of the width of this area if we consider Eq. (4) with $m^{2}=0$ and substitute for $\sigma^{2}$ its average value $\sigma_{\mathrm{rms}}^{2}=\sigma^{2} / 2$. There are two time scales characterizing the solutions of this equation. The first one is related to the friction term and is given by $t_{H}^{-1}=3 H / 2$. For $\sigma \ll M$ and $\phi / M \ll \sqrt{\lambda / g} M / \sigma$, we have

$$
t_{H}=\sqrt{\frac{2}{3 \pi \lambda}} \frac{M_{\mathrm{Pl}}}{M^{2}} .
$$

The other time scale is obtained if we neglect the friction term and consider the oscillations of the $\phi$ field. One-fourth of the period gives the typical time for the system to roll to the origin and away from an inflationary solution. It is given by

$$
t_{\mathrm{osc}}=\frac{\pi}{\sqrt{2 g}} \frac{1}{\sigma} .
$$

Inflation sets in if $t_{\mathrm{osc}}>t_{H}$, which gives

$$
\frac{\sigma}{M_{\mathrm{Pl}}}<\sqrt{\frac{3 \pi^{3} \lambda}{4 g}}\left(\frac{M}{M_{\mathrm{Pl}}}\right)^{2} .
$$

For comparable couplings $\lambda, g$, our assumptions for the derivation of the above bound break down when either $M$ $\sim M_{\mathrm{Pl}}$ or $\phi \gtrsim \sqrt{4 / 3 \pi^{3}} M_{\mathrm{Pl}} \simeq 0.2 M_{\mathrm{Pl}}$. This approximate analysis is verified by the results in Figs. 3 and 7. We have also checked Eq. (17) numerically for $M=10^{-3} M_{\mathrm{Pl}}$. For this value, the large continuous range of inflationary initial conditions moves to $\phi$ field values above $M_{\mathrm{Pl}}$.

The implications for the initial configuration that will lead to the onset of inflation are severe. For initial field values 
below $M_{\mathrm{Pl}}$ and $M \lesssim 10^{-3} M_{\mathrm{Pl}}$, the most favorable area of inflationary initial conditions is around the $\phi$ axis. Throughout a region of space the size of the order of the Hubble length (which initially is $\sim M_{\mathrm{Pl}}^{-1}$ ), the spatial variation of the $\sigma$ field in Planck units cannot exceed the RHS of Eq. (17). If this condition is not satisfied, the fields in different parts of the original homogeneous space region will evolve towards very different values. In one part they may end up in the valleys along the $\phi$ axis, while in another they may settle at the minima of the potential. Before inflation, the size of space regions shrinks compared to the Hubble distance (for a quantitative discussion, see below). As a result, large inhomogeneities are expected at scales smaller than $\sim H^{-1}$ when the evolution of the fields finally slows down. These will prevent the onset of inflation. We conclude that, for realistic inflationary models (that require $M \lessgtr 10^{-3} M_{\mathrm{Pl}}$ ), a significant fine tuning is required for the initial configuration that will lead to inflation.

The argument that we have presented is not rigorous. In principle, one should integrate the field equations for a configuration with a small spatial dependence and observe the growth of the perturbation [16]. However, we believe that the approximation of considering the initial space region as being composed of a few smaller regions with slightly different constant field values captures the essence of the problem. For this reason, we expect that a more rigorous treatment will not invalidate our qualitative conclusions.

Up until now we have not considered the kinetic energy density of the fields. We have assumed that the fields evolve from a homogeneous value with zero time derivatives. This is not a good approximation when the potential is much smaller than $M_{\mathrm{Pl}}^{4}$. A more reasonable approximation is to fix the total initial energy density at $M_{\mathrm{Pl}}^{4}$, and consider a kinetic term equal to the difference between the total energy density and the potential. However, this does not fix the direction of the initial time derivative of the fields. If this direction is uniform throughout the initial space region, we do not expect a qualitative change in the behavior we have observed previously [16]. In Fig. 8 we display an array of initial field values in Planck units for a theory with $M=10^{-2} M_{\mathrm{Pl}}$. The initial energy density has been fixed at $E=10^{-4} M_{\mathrm{Pl}}^{4}$. The initial time derivatives of the fields have been chosen equal and positive. We observe a strong similarity between Figs. 6 and 8 . This is explained by the fact that the initial time derivative quickly causes the displacement of the fields from the $\phi$ axis into an area where $\phi, \sigma \sim 0.1 M_{\mathrm{Pl}}$. There, the pattern of Fig. 6 is expected. The larger inflationary strip of Fig. 7 has disappeared. If the initial energy density is taken $E$ $\sim M_{\mathrm{Pl}}^{4}$, the fields are displaced to regions $\phi, \sigma \sim M_{\mathrm{Pl}}$, where the inflationary strips are narrower than the ones depicted in Fig. 8. We conclude that the presence of an initial kinetic term imposes much stronger constraints on the initial conditions than that of Eq. (17). There is the exception of an initial time derivative along the $\phi$ axis. However, this possibility leads to field values larger than $M_{\mathrm{Pl}}$. The variation of the direction of the time derivative within the initial space region results in more irregular patterns than that of Fig. 8, and strengthens the constraints further.

We have also followed the evolution of the scale factor $R$ relative to the Hubble parameter, starting from an initial

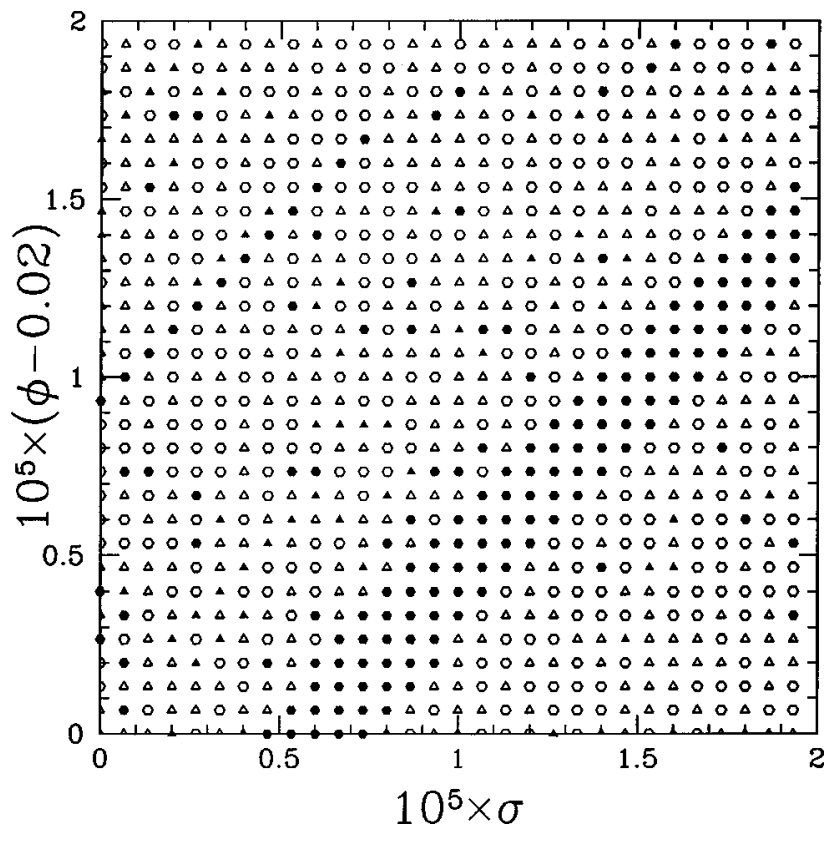

FIG. 8. $M=10^{-2} M_{\mathrm{Pl}}, \lambda=1, g=1$. Initial total energy density $E=10^{-4} M_{\mathrm{Pl}}^{4}$ and $\dot{\phi}=\dot{\sigma}>0$.

value $R_{0} \sim H_{0}^{-1}$. For the initial conditions of Fig. 8 that lead to inflation, we find that at the onset of inflation $R^{-1}$ is typically smaller than $H^{-1}$ by a factor of order 10 . For initial energy densities $E \sim M_{\mathrm{Pl}}^{4}$, this factor becomes of order 100 . This implies that the initial homogeneous region should extend far beyond a few initial Hubble lengths for this region to inflate. This factor becomes of order 1 if the initial time derivative of the fields is small and the fields start near the $\phi$ axis and move quickly on it.

We have based our discussion on the integration of the classical equations of motion. The presence of quantum (or thermal) fluctuations of the fields around their average values can be treated as a stochastic noise. We expect that this contributes an additional destabilizing factor, as it can easily knock the fields off an inflationary trajectory and enhance the irregularity of patterns in Figs. 1-8.

We conclude that hybrid inflation with the potential of Eq. (1) is not "natural." An extremely homogeneous initial field configuration must be assumed for initial field values below $M_{\mathrm{Pl}}$. For realistic models with $M \leqslant 10^{-3} M_{\mathrm{Pl}}$, the $\sigma$ field must be homogeneous with at least $\sim 10^{-6}-10^{-5}$ accuracy. This bound may be strengthened by several orders of magnitude if the initial fields have a significant time derivative. These constraints do not apply if the initial values of the fields are larger than $M_{\mathrm{Pl}}$. One could imagine that inflation starts in this region and continues as the system rolls down the valley on the $\phi$ axis. It is not possible, however, to construct reliable field-theoretical models in the field range above $M_{\mathrm{Pl}}$.

Our results have direct application to the simplest supersymmetric model that supports hybrid inflation. It is described by the superpotential $[2,3]$

$$
W=\Phi\left(-\mu^{2}+\lambda \bar{\Psi} \Psi\right)
$$

Here $\Phi, \Psi$ and $\bar{\Psi}$ are chiral superfields, for which we assume canonical kinetic terms. The above superpotential is 
the only renormalizable one consistent with a continuous $\mathrm{U}(1) R$-symmetry under which $W \rightarrow e^{i \theta} W, \Phi \rightarrow e^{i \theta} \Phi, \bar{\Psi} \Psi$ $\rightarrow \bar{\Psi} \Psi$. The superfields $\Psi$ and $\bar{\Psi}$ transform under an internal gauge symmetry, while the $\Phi$ superfield is a gauge singlet. The gauge symmetry is spontaneously broken at the scale $\mu / \sqrt{\lambda}$. The scale of inflation is set by $\mu$ and is constrained by the COBE observations to be two or three orders of magnitude below $M_{\mathrm{Pl}}$.

The potential of the scalar components of the superfields (for which we use the same notation) is given by

$$
V=\left|-\mu^{2}+\lambda \bar{\Psi} \Psi\right|^{2}+\lambda^{2}|\Phi|^{2}\left(|\Psi|^{2}+|\bar{\Psi}|^{2}\right)+D \text { terms. }
$$

Vanishing of the $D$ terms is achieved along the $D$-flat directions where $|\Psi|=|\bar{\Psi}|$. If the fields are assumed real, the potential along the $D$-flat directions is completely analogous to that of Eq. (1) but without a mass term for $\Phi$. The slope along the inflationary trajectory can be generated either through the addition of a soft supersymmetry-breaking mass for $\Phi$ [2] or by logarithmic radiative corrections [3]. Our conclusions about the fine-tuning of the initial configuration that will lead to the onset of inflation are independent of the term providing this slope. The Figs. 1-8 were generated for zero slope, and the constraints that we derived are strengthened if a significant slope is present. Including the imaginary parts of the scalar fields (some of which can be eliminated through appropriate gauge and $R$ transformations along the $D$-flat directions) does not alter the qualitative picture.

It must be pointed out, however, that our conclusions do not automatically apply to the models with superpotentials different from that of Eq. (18). Models such as the smooth hybrid inflation of Ref. [5] may display less sensitivity on the initial conditions and their separate investigation is necessary.

We would like to thank J. Garcia-Bellido, G. Lazarides, and C. Panagiotakopoulos for many useful discussions.
[1] A. D. Linde, Phys. Lett. B 259, 38 (1991); Phys. Rev. D 49, 748 (1994).

[2] E. J. Copeland, A. R. Liddle, D. H. Lyth, E. D. Stewart, and D. Wands, Phys. Rev. D 49, 6410 (1994).

[3] G. Dvali, Q. Shafi, and R. Schaefer, Phys. Rev. Lett. 73, 1886 (1994).

[4] E. D. Stewart, Phys. Rev. D 51, 6847 (1995).

[5] G. Lazarides and C. Panagiotakopoulos, Phys. Rev. D 52, 559 (1995)

[6] C. Panagiotakopoulos, Phys. Rev. D 55, 7335 (1997); Phys. Lett. B 402, 257 (1997).

[7] P. Binétruy and G. Dvali, Phys. Lett. B 388, 241 (1996); E. Halyo, ibid. 387, 43 (1996).

[8] A. D. Linde and A. Riotto, Phys. Rev. D 56, 1841 (1997).

[9] S. Dimopoulos, G. Dvali, and R. Rattazzi, Phys. Lett. B 410, 119 (1997).
[10] G. Lazarides, C. Panagiotakopoulos, and N. D. Vlachos, Phys. Rev. D 54, 1369 (1996).

[11] G. Lazarides and N. D. Vlachos, Phys. Rev. D 56, 4562 (1997).

[12] A. D. Linde, Particle Physics and Inflationary Cosmology (Harwood Academic, Chur, Switzerland, 1990), and references therein.

[13] A. R. Liddle and D. H. Lyth, Phys. Rep. 231, 1 (1993).

[14] S. W. Hawking, Phys. Lett. 115B, 295 (1982); A. A. Starobinsky, ibid. 117B, 175 (1982); A. H. Guth and S.-Y. Pi, Phys. Rev. Lett. 49, 1110 (1982); D. H. Lyth, Phys. Lett. 147B, 403 (1984); 150B, 465 (1985); Phys. Rev. D 31, 1792 (1985).

[15] D. H. Lyth, LANCS-TH/9614, hep-ph/9609431.

[16] D. S. Goldwirth and T. Piran, Phys. Rep. 214, 223 (1992). 